\title{
An IP Passing Protocol for Vehicular Ad Hoc Networks with Network Fragmentations
}

\author{
Yuh-Shyan Chen \\ Department of Computer Science \\ and Information Engineering \\ National Taipei University \\ Taipei, Taiwan \\ Email: yschen@mail.ntpu.edu.tw
}

\author{
Chih-Shun Hsu \\ Department of \\ Information Management \\ Shih Hsin University \\ Taipei, Taiwan \\ Email: cshsu@cc.shu.edu.tw
}

\author{
Wei-Han Yi \\ Graduate Institute of \\ Communication Engineering \\ National Taipei University \\ Taipei, Taiwan \\ Email: weihan.yi@gmail.com
}

\begin{abstract}
When a vehicle is moving fast in a highway, how to effectively reduce the handoff delay and maintain the connectivity of the vehicle to the Internet is an important issue. The existing IP passing protocol may be able to reduce the handoff delay and maintain the connectivity of the vehicle to the Internet when all the vehicles are connected. However, when the vehicle density is low or the speeds of vehicles are varied, the vehicle may not be able to communicate with the intended vehicle either directly or through multi-hop relays because of network fragmentation. Hence, when network fragmentation occurs, a vehicle cannot pass its IP address to the intended vehicle through existing IP passing protocols and thus incurs longer handoff latency and higher packet loss rate, and these would lower down the throughput of the network. To improve existing IP passing protocols, we propose an IP passing protocol for VANETs with network fragmentations. The proposed protocol can postpone the time to release IP addresses to the DHCP server (extend IP lifetime) and select a faster way to get the vehicle's new IP address. During the extended IP lifetime, the vehicle can acquire an IP address through multi-hop relays from the vehicles on the lanes of the same or opposite direction, and thus reduces handoff delay and maintain the connectivity to the Internet. Simulation results have shown that the proposed scheme is able to reduce IP acquisition time, handoff latency, packet loss rate, and extend IP lifetime.
\end{abstract}

Index Terms-Vehicular Ad Hoc Network (VANET), IP passing, Mobility Management, Wireless Network, Network Fragmentation.

\section{INTRODUCTION}

Due to the advancement of wireless communication technology, users may enjoy many kinds of services through Internet ubiquitously. As the wireless transmission rate increases, users can make a VoIP call, browse website, download data, watch $\mathrm{TV}$, and get road traffic information or real-time weather report from Internet through wireless communications.

Since every vehicle can be equipped with a short or medium-range wireless transceiver, vehicles may communicate with each other. Vehicles may acquire information and services through the V2V (Vehicle-to-Vehicle) or I2V (Infrastructure-to-Vehicle) communications. The V2V communication is based on the dedicated short range communications (DSRC) technology; while the V2I communication is based on GPRS/3G, Wi-Fi or WiMAX. In VANETs, since the moving speed of a vehicle is so high that it is hard to maintain a seamless handoff and stable connectivity to the Internet. To achieve a seamless handoff for an IP-based communication, the IP of the mobile device must be assigned and reassigned efficiently.
The Mobile Internet Protocol version 4 (MIPv4) [1] has been proposed by the Internet Engineering Task Force (IETF). Since Mobile Internet Protocol version 4 (MIPv4) may face problems like the shortage of IP address and the weak security mechanism, MIPv6 [2] is proposed to alleviate the above problems. To improve the efficiency of MIPv6, several schemes have been proposed, namely Fast Mobile IPv6 (FMIPv6) [3], Proxy Mobile IPv6 (PMIPv6) [4] and Hierarchical Mobile IPv6 (HMIPv6) [5].

Mobile IP has an important characteristic that it configures the IP address by neighbor discovery or auto-configuration. There are two auto-configuration mechanisms: the stateful and stateless mechanisms. Dynamic Host Configuration Protocol (DHCP) [6] which has been adopted in both IPv4 and IPv6, is a stateful auto-configured mechanism that each IP address is auto-configured and managed by a DHCP server [7]. The stateless auto-configured mechanism is adopted by IPv6, which creates link-local IPv6 address. Addressing in vehicular networks could be achieved by using DHCP, which has been extensively used in computer networks. The DHCP process often requires a lot of time to complete the association and it may consume too much time to achieve a seamless handoff. Hence how to reduce the time for acquiring a new IP address is an important issue.

Several schemes for VANETs have been proposed [8] [9] [10] [11] [12] [13] [14] so as to keep the mobile devices connected to the Internet and reduce the complexity of handoff procedure. However, they do not consider the impacts of network fragmentations. When a vehicle is leaving the communication region of its serving Base Station (BS) and is moving to the boundary of the target BS's communication region, it may acquire a new IP address through IP passing [8] [12] from the vehicles on the lanes of the same or opposite direction so as to reduce handoff delay. However, when the vehicle density is low or the speeds of vehicles are varied, the vehicle may not be able to communicate with the intended vehicle either directly or through multi-hop relays because of network fragmentation. Hence, when network fragmentation occurs, a vehicle cannot pass its IP to the intended vehicle through existing IP passing protocols and thus incurs longer handoff latency and higher packet loss rate.

To improve existing IP passing protocols [8] [12], we propose an IP passing protocol for VANETs with network fragmentations. In the proposed protocol, when a vehicle is going to leave the target BS and network fragmentation occurs, although the vehicle can not pass its IP address to the intended vehicle, it still will pass its IP address to the vehicle that remains in the target $\mathrm{BS}$ and thus postpones the time to release its IP address to the DHCP server (extend IP lifetime). During 


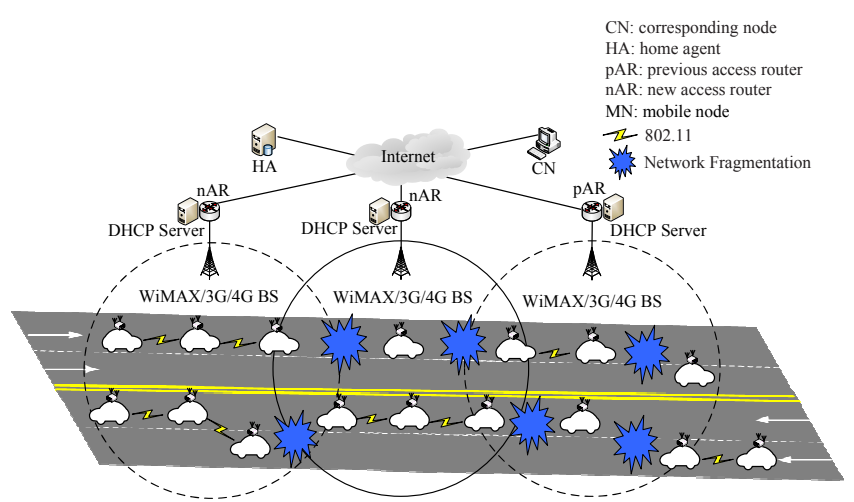

Fig. 1. System architecture.

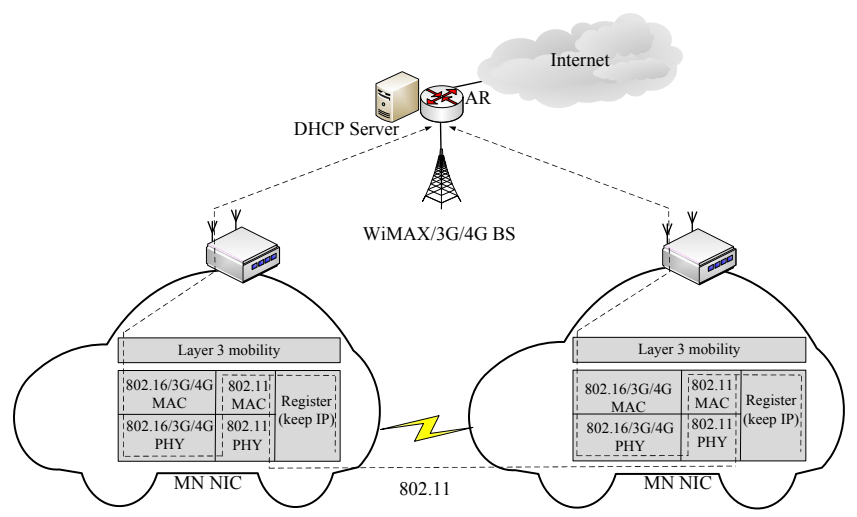

MN NIC: Mobile node Network Interface Card

Fig. 2. Protocol stacks the mobile node.

the extended IP lifetime, as a vehicle is going to enter the target $\mathrm{BS}$, it can acquire an IP address through multi-hop relays from the vehicle which carries the released IP address, and thus it can reduce the handoff delay and maintain the connectivity to the Internet. Simulation results have shown that the proposed scheme is able to reduce IP acquisition time, handoff latency, packet loss rate, and extend IP lifetime.

The remainder of this paper is organized as follows. Section 2 overviews the system architecture, and the basic ideas of the proposed schemes. Section 3 describes the proposed IP passing protocol. Performance evaluation is presented in section 4. Section 5 concludes this paper.

\section{Preliminaries}

In this section, we first describe the assumptions and system architecture, and then we explain the challenges and basic ideas of this work.

\section{A. System Architecture}

The system architecture of our protocol is shown in Fig. 1. The proposed protocol is design for VANETs with network fragmentations in a highway with two lanes in each direction. We assume that there are base stations (BS) (e.g. WiMAX, 3G or $4 \mathrm{G} \mathrm{BS}$ ) scattered along the roadside. The home agent (HA) records the vehicle's new location and the correspondence node $(\mathrm{CN})$ serves as a remote server e.g. FTP or Web server etc. Each vehicle, regarded as a mobile node $(\mathrm{MN})$, is equipped with two communication interfaces, one for communicating with the BS (e.g. WiMax, 3G or 4G Bs) and one for communicating with other vehicles. Each vehicle can connect to the Internet via WiMAX (or $3 \mathrm{G} / 4 \mathrm{G}$ ) interface and can

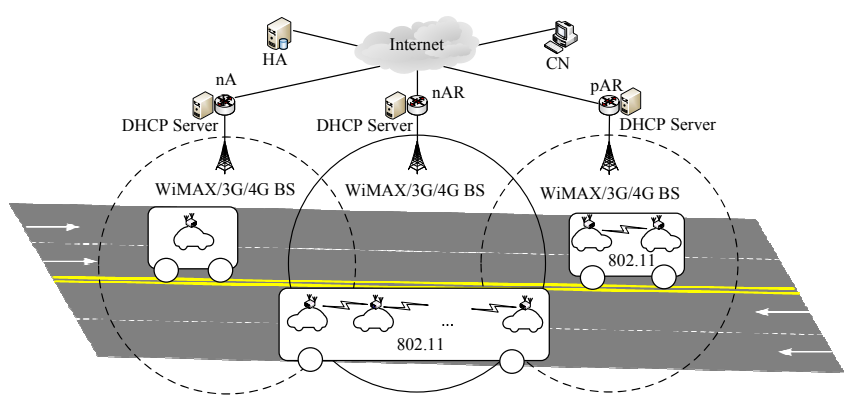

Fig. 3. Forming virtual buses in VANETs

communicates with other vehicles via IEEE 802.11 (Wi-Fi or DSRC) interface. Fig. 2 shows the protocol stack of the mobile node. In addition, each vehicle is equipped with registers to keep the IP address of the serving BS's communication region, location obtained by GPS, driving speed and other related information. Two or more neighboring vehicles on the lanes of the same or opposite direction may be form as a virtual bus [12], which may acquire or pass IP addresses by the cooperation of the vehicles in the virtual bus as shown in Fig. 3.

\section{B. Basic idea and challenges}

On the highway, when a vehicle moves to a new subnet, the vehicle will receive broadcast packet from the target BS and most importantly perform the handoff procedure. The traditional handoff procedure includes two parts, the layer 2 (link layer) and layer 3 (network layer) handoff procedures. The handoff procedure contains signal measurement, network layer movement detection, DAD procedure and registration. The DAD procedure is time consuming and thus will cause the link to be disconnected.

On the highway, since the vehicle is moving so fast that the handoff latency must be reduced. Hence, how to acquire IP addresses immediately is a very important issue. The concepts of IP passing [8] and virtual bus [12] are adopted in our protocol so as to reduce the time to acquire a new IP address. However, the vehicle may not be able to acquire its new IP address through IP passing and virtual bus because of network fragmentation. To solve the problem, two strategies have been adopted in the proposed protocol. First, when a vehicle is going to leave the target BS and network fragmentation occurs, although the vehicle can not pass its IP address to the vehicle that is going to enter the target BS, still it will pass its IP address to the vehicle that remains in the target BS and thus postpones the time to release its IP address to the DHCP server (extend IP lifetime). Second, during the extended IP lifetime, as a vehicle is going to enter the target $\mathrm{BS}$, it can acquire an IP address through multi-hop relays from the vehicle which carries the released IP and thus provide more chances for a vehicle to acquire a new IP address through IP passing.

\section{THE PROPOSED IP PASSING PROTOCOL}

In this section, we first show the flowchart of the proposed protocol, then we describe the two phases of the proposed protocol. For the ease of describing the proposed protocol, we define the following notations as shown in Table I:

- LMN: the vehicle which is going to leave the serving BS.

- KMN: the vehicle which keeps the IP released by LMN.

- EMN: the vehicle which is going to enter the target BS.

The handoff procedure contains phases as follows, the information collecting phase, the fast IP acquiring phase, the cooperation of mobile node (vehicle) phase, the make 
Table I: Definition of notations

\begin{tabular}{|l|l|}
\hline Notation & Definition \\
\hline & The vehicle (mobile node) receives and keeps the passed IP. \\
\hline & The vehicle (mobile node) leaves target BS's communication region. \\
\hline
\end{tabular}

before break phase, and the route redirection phase. In the information collecting phase, each mobile node (vehicle) will broadcast its own and its neighboring vehicles' locations, moving speeds, and directions periodically. Besides, it should also rebroadcast the messages it received according to the TTL (time to live) of the messages to determine the IP passing direction. If the TTL of message is greater than 0 , then the message should be rebroadcast. The TTL is set according to the intended size of the virtual bus and the communication range of the base station. After collecting the information of the nearby vehicle, a vehicle can realize the neighboring vehicle' locations, moving speeds, and directions and thus can group proper vehicles to form a virtual bus and can select proper cooperative vehicles to assist it to pass IP address at the proper moment.

Fig. 4 shows the flowchart of the proposed IP passing protocol, which contains two phases, the extension of IP lifetime phase and the IP acquisition phase.

\section{A. Extension of IP lifetime}

As shown in Fig. 4, when LMN is going to leave the target BS's communication region, if LMN can communicate with EMN, it follows the existing IP passing protocol to pass its IP address to EMN. However, if LMN cannot pass its IP address to EMN due to network fragmentation, LMN will pass its IP address to KMN through the virtual bus as shown in Fig. 5, such that LMN does not need to release its IP address to the DHCP server. Instead, its IP address is kept in KMN so that the IP address will have more chances to be passed to EMN. If there is no vehicle behind LMN that is on the lanes of the same direction, LMN will try to pass its IP address to the vehicle that is on the lanes of the opposite direction, as shown in Fig. 6. The IP address can also be passed to the vehicle on lanes of both directions through the cooperation of vehicles on the lanes of different directions as shown in Fig. 7. If LMN cannot find any proper KMN, it will release its IP address via the DHCP server.

Note that, when KMN receives the IP passing packet from LMN, it will reply a keep IP ACK packet to LMN and keep LMN's IP address. After LMN receives the keep IP ACK packet, it will break the connection with the target BS. As time goes by, KMN may approach the border of target BS's communication region, it will become LMN and try to pass the IP address to EMN or other KMN.

\section{B. IP acquisition and handoff procedure}

In VANETs, how to reduce the IP acquisition time is a very important issue. As EMN enters the target BS's communication region, EMN still connects to Internet via IEEE 802.16 (WiMAX) with previous access router ( $\mathrm{pAR}$ ) and performs the following IP acquisition procedure as shown in Fig. 8.

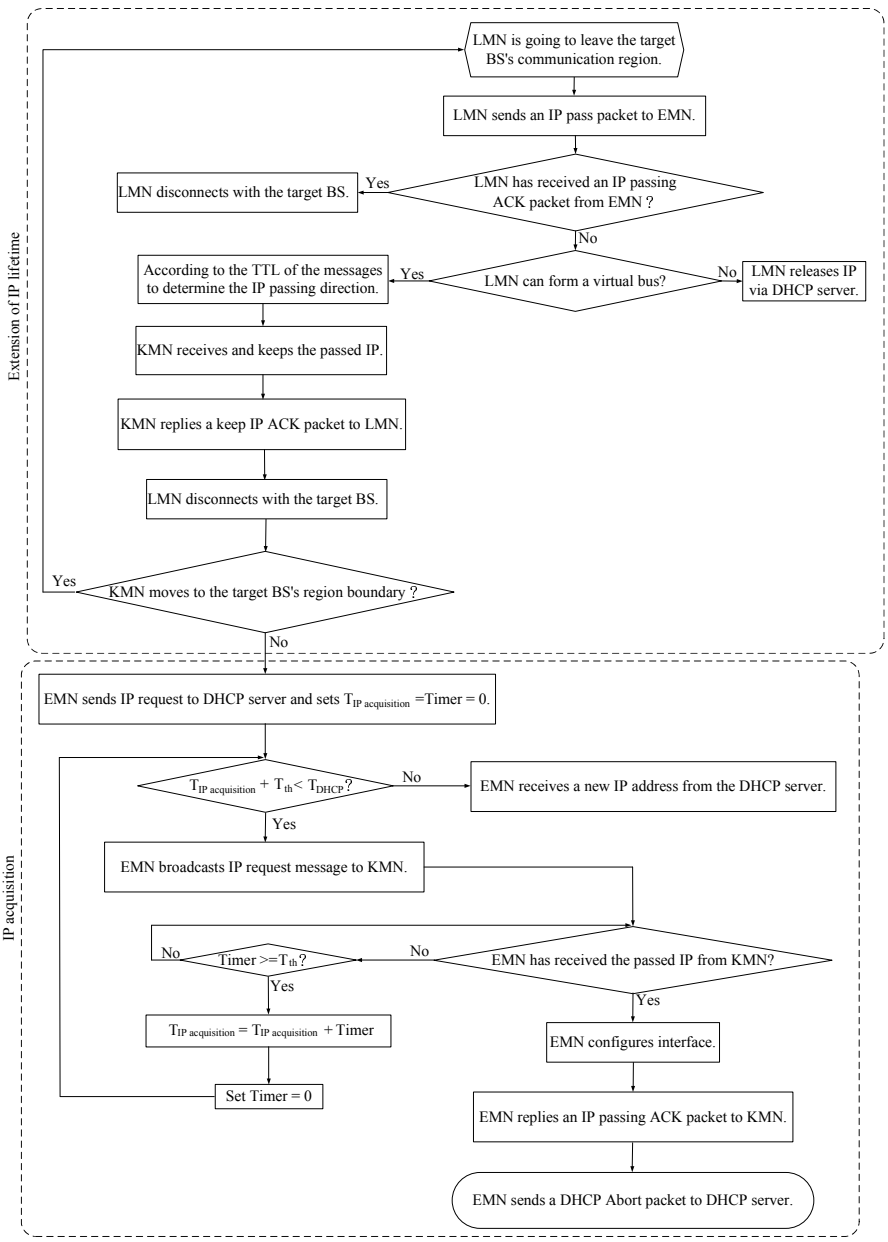

Fig. 4. The flowchart of our scheme.

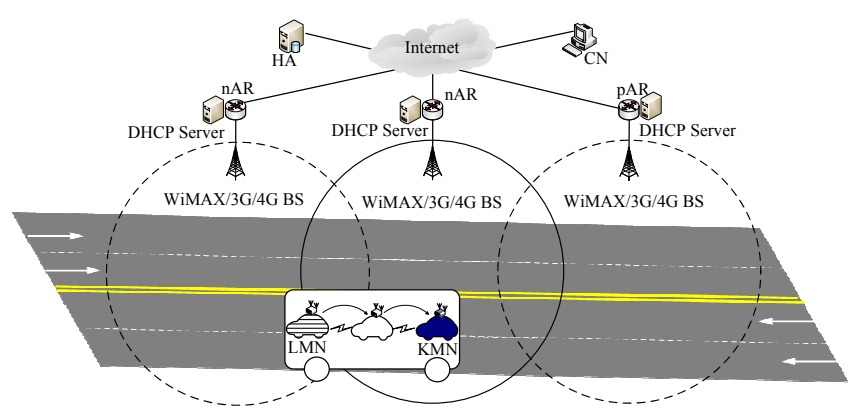

Fig. 5. LMN passes its IP address to KMN which is on the lanes of the same direction.

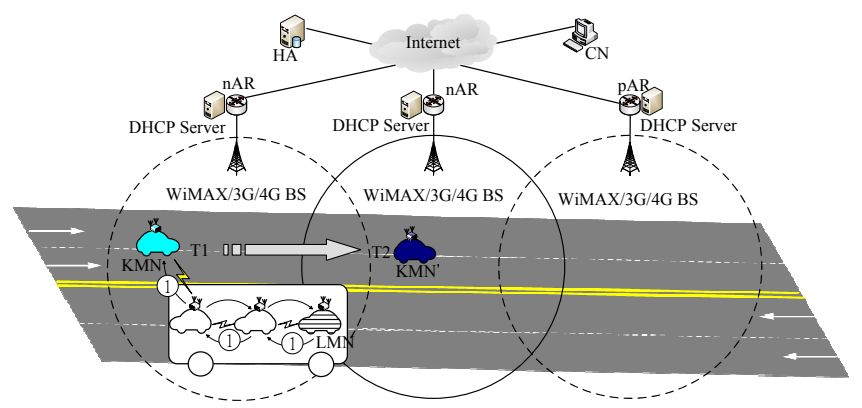

Fig. 6. LMN passes its IP address to KMN which is on the lanes of the opposite direction. 


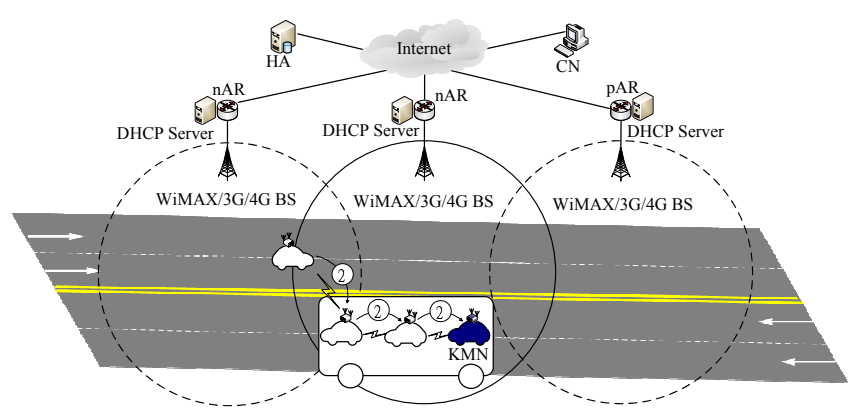

Fig. 7. LMN passes its IP address to KMN through the cooperation of the vehicles on the lanes of both directions.

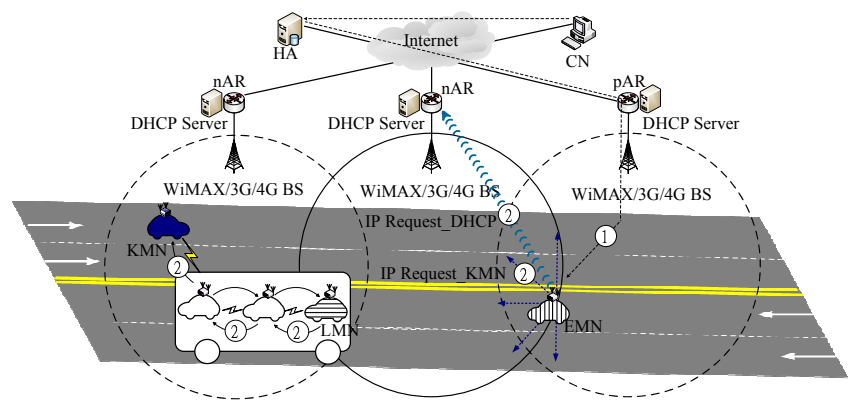

Fig. 8. IP acquisition and handoff procedure.

S1: EMN broadcast an IP request packet to KMN and sets its timer and $T_{\text {acquisition }}$ as 0 , where $T_{\text {acquisition }}$ is the time that EMN has already spent on acquiring an IP address from KMN. At the same time, it sends an IP request packet to the DHCP server.

S2: EMN selects a faster way to acquire a new IP address: When $T_{\text {acquisition }}+T_{t h} \geq T_{D H C P}$, it indicates that EMN can receive an IP address from the DHCP server earlier than acquiring an IP address from KMN, where $T_{t h}$ is the amount of time for EMN to send an IP request packet to $\mathrm{KMN}$ and receive an IP address from $\mathrm{KMN}$; while $T_{D H C P}$ is the amount of time for EMN to send an IP request packet to the DHCP server and receive an IP address from the DHCP server. Under such condition, EMN will not broadcasts an IP request packet to KMN. If Timer $\geq T_{t h}$ and EMN has not received an IP address from KMN, it indicates that the IP request packet sent by EMN or the IP address sent by KMN is lost. Under such condition, EMN will set $T_{\text {acquisition }}$ as $T_{\text {acquisition }}+$ Timer, and then reset its timer as 0 and check if there is still enough time to get an IP address from KMN. If the remaining time is enough (e.g. $T_{\text {acquisition }}+T_{t h}<$

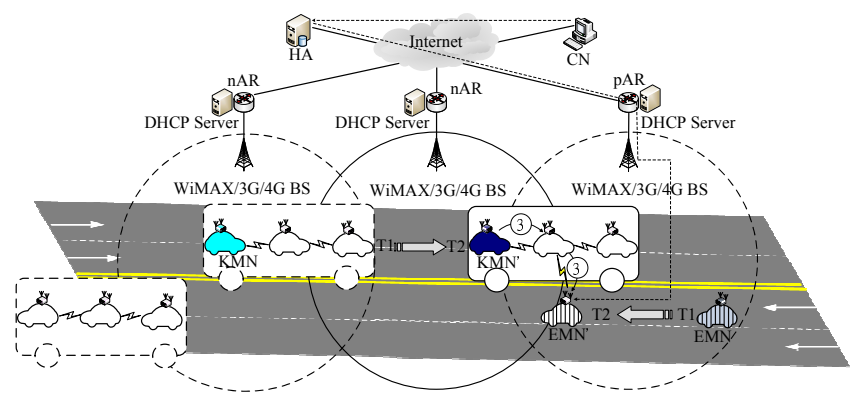

Fig. 9. EMN acquires an IP address from the vehicles on the lanes of the opposite direction.

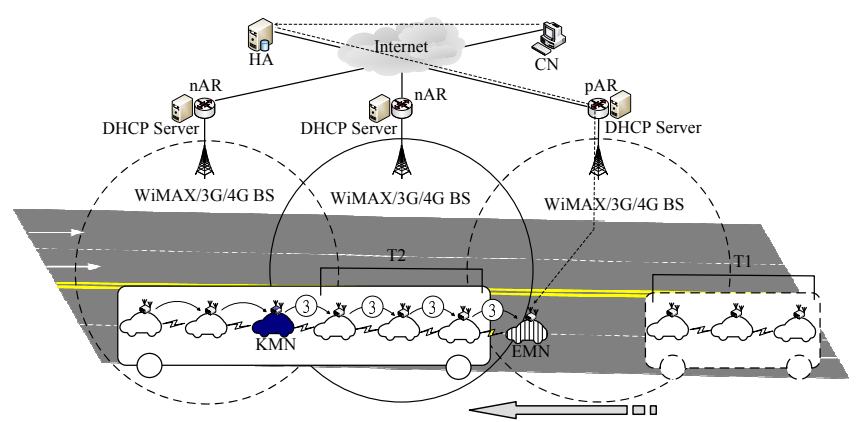

Fig. 10. EMN acquires an IP address from the vehicles on the lanes of the same direction.

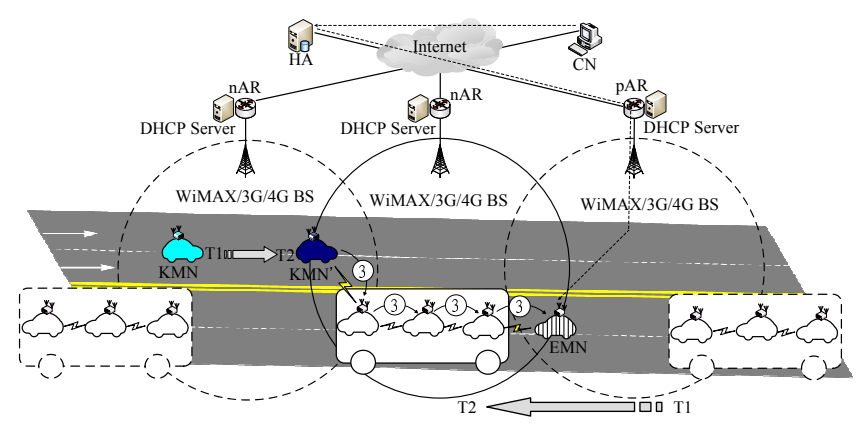

Fig. 11. EMN acquires an IP address via the cooperation of vehicles on the lanes of both directions.

$\left.T_{D H C P}\right)$, EMN will rebroadcast the IP request packet, if the remaining time is not enough, EMN will wait for the DHCP server to assign an IP address.

Fig. 9 and 10 show how EMN acquires an IP address from KMN which is on the lanes of the opposite and the same directions, respectively. During time T1 to time T2, EMN moves from the serving BS to the target BS's communication region. EMN can get an IP address from KMN, because LMN has passed its IP address to KMN which is on the opposition direction, KMN keeps the IP address and then gets the chance to pass the IP address to EMN. EMN can also acquires its IP address from the vehicles on the lanes of both directions as shown in Fig. 11.

After EMN acquired an IP address, EMN performs the binding update and the bidirectional tunnelling process.

The message flow diagram of the proposed IP passing protocol is shown in Fig. 12.

\section{Mathematical Analysis}

To evaluate the performance of our protocol, we use Markov chains to analyze the average IP acquisition time and the probability that KMN passes an IP address to EMN successfully of the proposed protocol. Two scenarios are considered in our analysis, the first scenario considers only the vehicles on the lanes of the same direction as shown in Fig 13; while the second scenario considers the vehicles on the lanes of both directions as shown in Fig 14.

The notations used in the first scenario are defined as follows:

- $m$ : the maximum possible number of vehicles which are on the lanes of the same direction and between KMN and EMN.

- $R_{i}^{S}$ : is the ID of the $i$-th vehicle which is on the lanes of the same direction with KMN and is between KMN and EMN, where $1 \leq i \leq m$. 


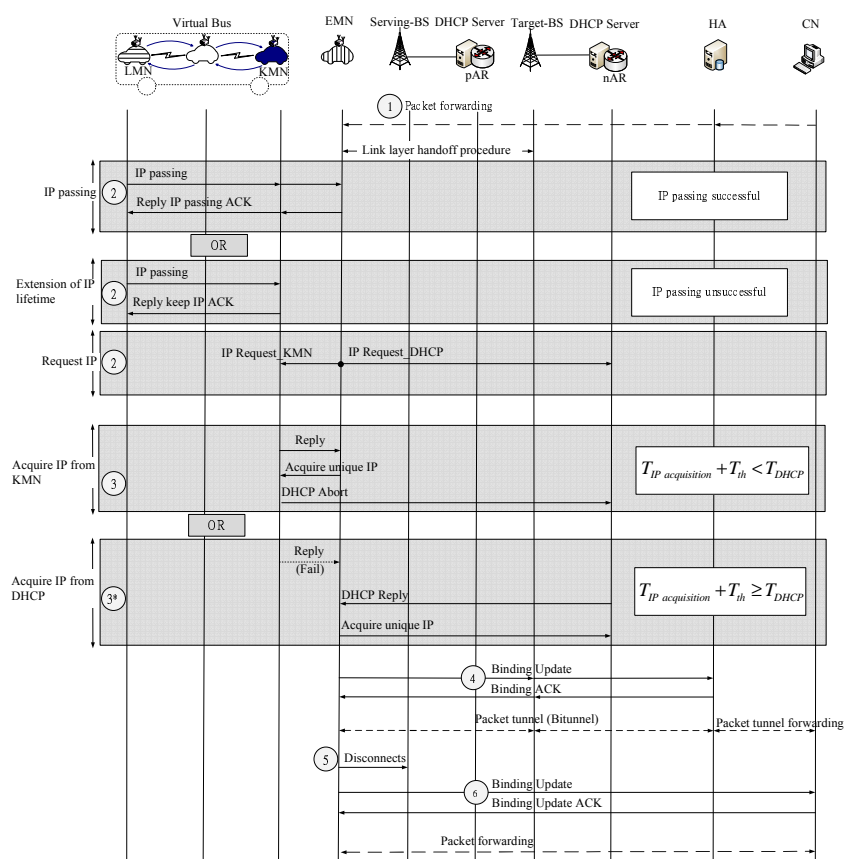

Fig. 12. The message flow diagram of IP passing protocol and handoff procedure.

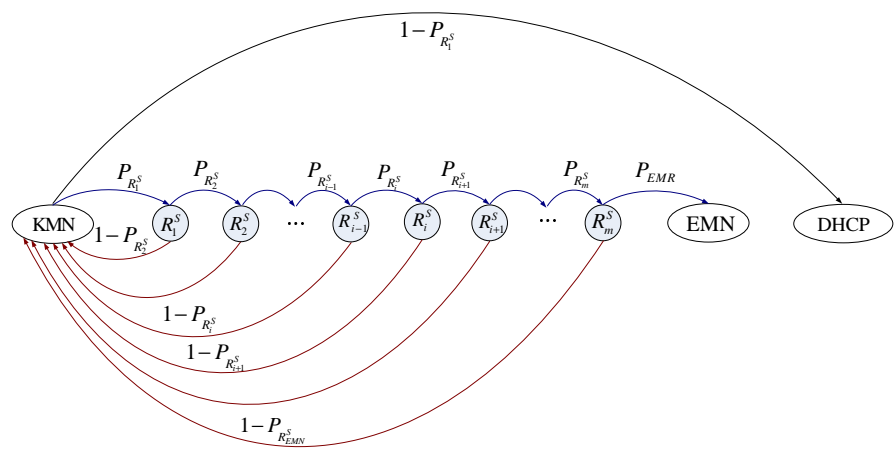

Fig. 13. Markov chains of the scenario that there are vehicles on the lanes of same direction.

- $P_{R_{i}^{S}}$ : is the probability that the IP address kept in KMN can be passed from vehicle KMN to $R_{i}^{S}$ successfully if $i=1$, or the probability that the IP address kept in KMN can be passed from $R_{i-1}^{S}$ to $R_{i}^{S}$ successfully if $2 \leq i \leq m$ (e.g. the distance between two adjacent vehicles is smaller than their communication range).

- $P_{E M N}$ : is the probability that the IP address kept in KMN can be passed from $R_{m}^{S}$ to EMN successfully.

- $T_{S_{-} \text {average }}$ is the average IP acquisition time for EMN.

The probability that the IP address can be passed from KMN to the $i$-th vehicle is

$$
\prod_{a=1}^{i} P_{R_{a}^{S}}
$$

The probability that the IP address can be passed from KMN through $i$ vehicles to EMN successfully (denoted as $P_{S_{-}}$pass $)$ is derived as Equation 1:

$$
P_{S \_p a s s}=P_{E M N} \prod_{a=1}^{i} P_{R_{a}^{S}}
$$

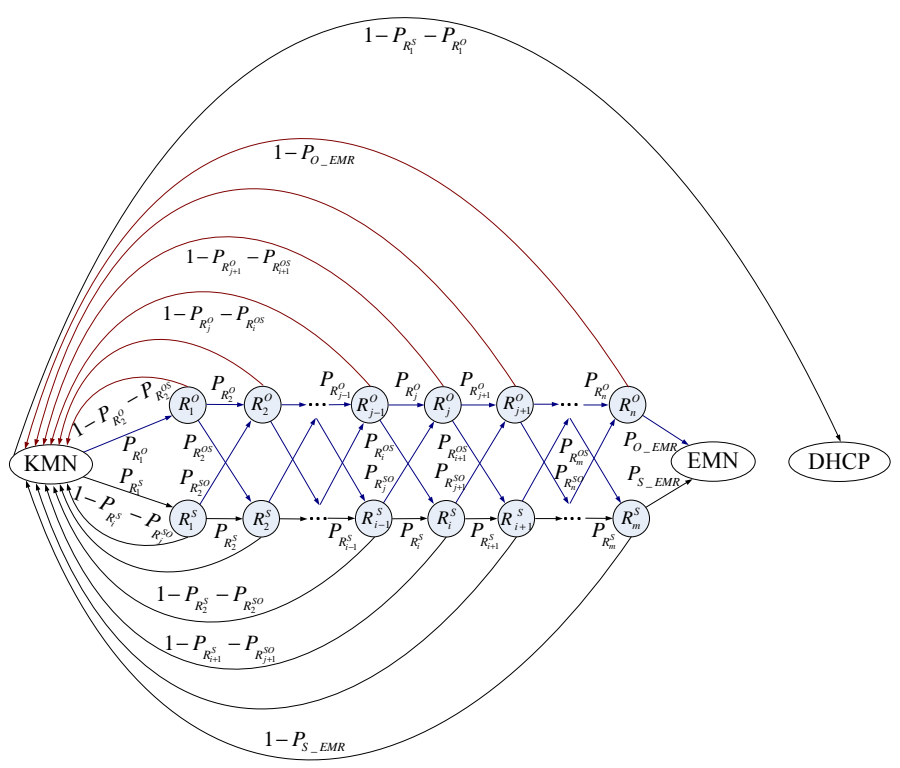

Fig. 14. Markov chains of the scenario that there are vehicles on the lanes of both directions.

EMN may acquire an IP address either from KMN or from the DHCP server. Therefore, the average IP acquisition time for EMN $T_{S_{-} \text {average }}$ is derived as Equation 2:

$$
T_{S \_a v e r a g e}=T_{S \_p a s s} \cdot P_{S \_p a s s}+T_{D H C P} \cdot\left(1-P_{S \_p a s s}\right)
$$

, where $T_{S \text { pass }}$ is the time required for passing an IP address from KMN to EMN through the vehicles on the same direction.

The additional notations used in the second scenario are defined as follows:

- $n$ : the maximum possible number of vehicles which are on the lanes of the opposite direction and between KMN and EMN.

- $R_{j}^{O}$ : is the ID of the $j$-th vehicle which is on the lanes of the opposite direction with KMN and is between KMN and EMN, where $1 \leq j \leq n$.

- $P_{R_{\text {OS }}}:$ is the probability that the IP address kept in KMN can be passed from vehicle $R_{j-1}^{O}$ to vehicle $R_{i}^{S}$ successfully, where $2 \leq i \leq m$.

- $P_{R_{j}^{O}}$ : is the probability that the IP address kept in KMN can be passed from vehicle $R_{j-1}^{O}$ to vehicle $R_{j}^{O}$ successfully, where $2 \leq j \leq n$.

- $P_{R_{j}^{S O}}:$ is the probability that the IP address kept in KMN can be passed from vehicle $R_{i-1}^{S}$ to vehicle $R_{j}^{O}$ successfully, where $2 \leq j \leq n$.

- $1-P_{R_{i+1}^{S}}-P_{R_{j+1}^{S O}}$ : is the probability that the IP address kept in KMN cannot be passed either from vehicle $R_{i}^{S}$ to vehicle $R_{i+1}^{S}$ or from vehicle $R_{i}^{S}$ to vehicle $R_{j+1}^{O}$ successfully, where $1 \leq i \leq m-1$ and $1 \leq j \leq n-1$.

- $1-P_{R_{j+1}^{O}}-P_{R_{i+1}^{O S}}$ : is the probability that the IP address kept in KMN cannot be passed either from vehicle $R_{j}^{O}$ to vehicle $R_{j+1}^{O}$ or from vehicle $R_{j}^{O}$ to vehicle $R_{i+1}^{S^{j}}$ successfully, where $1 \leq i \leq m-1$ and $1 \leq j \leq n-1$.

- $P_{S_{-} E M N}$ : is the probability that the IP address kept in KM̄N can be passed from vehicle $R_{m}^{S}$ to EMN successfully. 
- $P_{O_{E} E M N}$ : is the probability that the IP address kept in KM̄N can be passed from vehicle $R_{n}^{O}$ to EMN successfully.

- $T_{S O}$ average: is the average time that EMN acquires an IP address either from KMN through the vehicles on the lanes of both directions or from the DHCP server.

The probability that the IP address can be passed from KMN to the second vehicle $\left(R_{2}^{S}\right)$ on the lanes of the same direction with KMN (denoted as $P_{\text {all__ }}^{S}$ ) is derived as follows:

$$
P_{\text {all_ } R_{2}^{S}}=P_{R_{1}^{S}} \cdot P_{R_{2}^{S}}+P_{R_{1}^{O}} \cdot P_{R_{2}^{O S}}
$$

The probability that the IP address can be passed from KMN to the second vehicle $\left(R_{2}^{O}\right)$ on the lanes of the opposite direction with KMN (denoted as $P_{\text {all_}} R_{2}^{O}$ ) is derived as follows:

$$
P_{a l l_{-} R_{2}^{S}}=P_{R_{1}^{O}} \cdot P_{R_{2}^{O}}+P_{R_{1}^{S}} \cdot P_{R_{2}^{S O}}
$$

The probability that the IP address can be passed from KMN to the $i$-th vehicle $\left(R_{i}^{S}\right)$ on the lanes of the same direction with KMN (denoted as $P_{\text {all__}} R_{i}^{S}$ ) is derived as follows:

$$
P_{a l l \_R_{i}^{S}}=P_{a l l \_R_{i-1}^{S}} \cdot P_{R_{i}^{S}}+P_{a l l_{-} R_{i-1}^{O}} \cdot P_{R_{i}^{O S}}
$$

The probability that the IP address can be passed from KMN to the $j$-th vehicle $\left(R_{j}^{O}\right)$ on the lanes of the opposite direction with KMN (denoted as $P_{a l l_{-} R_{j}^{O}}$ ) is derived as follows:

$$
P_{a l l \_R_{j}^{O}}=P_{a l l \_R_{i-1}^{O}} \cdot P_{R_{i}^{O}}+P_{a l l_{-} R_{i-1}^{S}} \cdot P_{R_{i}^{S O}}
$$

We can use dynamic programming to calculate $P_{a l l} R_{i}^{S}$ and $P_{\text {all_}} R_{\dot{j}}^{O}$. The probability that the IP address can be passed from KMN to EMN successfully from both directions (denoted as $\left.P_{S O \_p a s s}\right)$ is derived as Equation 3:

$$
P_{S O \_p a s s}=P_{O_{-} E M N} \cdot P_{a l l_{-} R_{j}^{O}}+P_{S_{-} E M N} \cdot P_{a l l_{-} R_{i}^{S}}
$$

EMN may acquire an IP address either from KMN or from the DHCP server. Therefore, the average IP acquisition time for EMN $T_{S O \_a v e r a g e}$ is derived as Equation 4:

$T_{S O \_a v e r a g e}=T_{S O \_p a s s} \cdot P_{S O \_p a s s}+T_{D H C P} \cdot\left(1-P_{S O \_p a s s}\right)$ , where $T_{S O \_p a s s}$ is the time required for passing an IP address from KMN to EMN through the vehicles on both directions.

\section{Performance Evaluation}

To further evaluate the performance of our protocol, we simulate the original mobility support of IPv6 [2], the IP passing protocol [8] and our protocol on Network Simulator2 (ns-2) [15]. For the simplicity of describing the simulation results, the original mobility support in IPv6 is denoted as MIPv6, the IP passing for VANETs is denoted as IP passing, our protocol is denoted as our scheme, and the analyzed result of our scheme is denoted as our scheme-A. Each simulation result is derived from the average of 100 simulations. The performances metrics observed in our simulations are shown as follows:

- The IP acquisition time is defined as the interval from the time when EMN sends the IP acquisition packet to the time when EMN gets its IP address either from KMN or from the DHCP server.

- The IP lifetime is defined as the interval from the time when LMN intends to release its IP address to the time when the IP address is released back to the DHCP server.

- The Handoff latency is defined as the interval from the time when the last packet is received from the old BS to
Table II: Simulation parameters

\begin{tabular}{|l|l|}
\hline Network size & $5000 \mathrm{~m} \times 50 \mathrm{~m}$ \\
\hline Transmission range of WiMAX & $1000 \mathrm{~m}$ \\
\hline Transmission range of WLAN & $250 \mathrm{~m}$ \\
\hline Vehicle speed & $5 \sim 100 \mathrm{~km} / \mathrm{h}$ \\
\hline Number of vehicles & $5 \sim 100$ \\
\hline Network fragmentation ratio & $0.0 \sim 1.0$ \\
\hline Length of IP passing (hops) & $1 \sim 20$ \\
\hline Packet size & 512 bytes \\
\hline Packet rate & 200 packets $/ \mathrm{sec}$ \\
\hline Simulation Time & $200 \mathrm{sec}$ \\
\hline
\end{tabular}

the time when the first packet is received from the new BS.

- The Packet loss rate is defined as the number of missed packets divided by the number of transmitted packets.

- The Throughput is defined as the amount of received data per second.

- The Message overhead is the total number of IP-passing packets and the packets to discover CV-MH (cooperative vehicle mobile host).

The simulation parameters are shown in Table II.

\section{A. IP acquisition time}
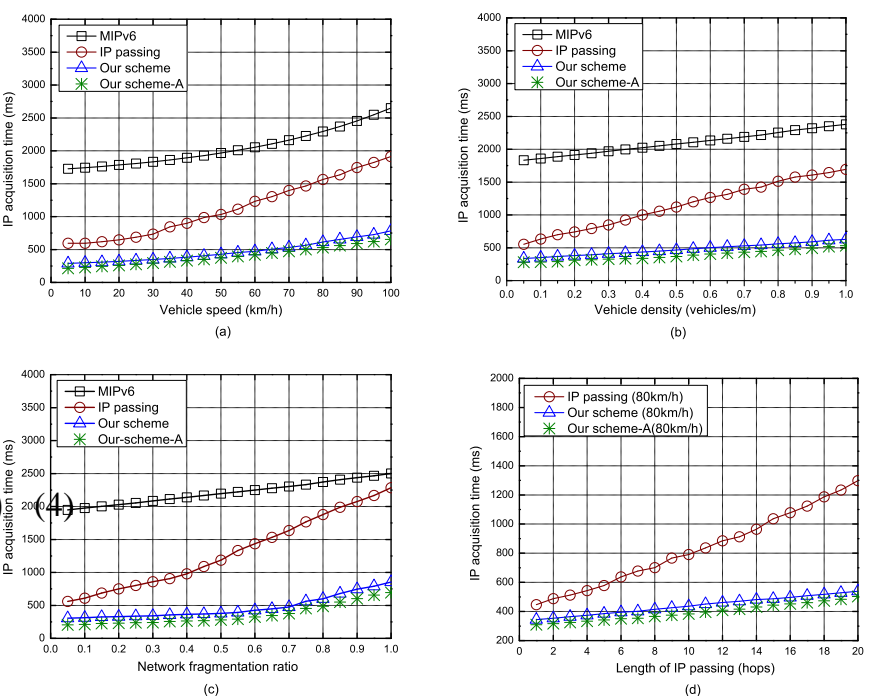

Fig. 15. (a) IP acquisition time vs. vehicle speed, (b) IP acquisition time vs. vehicle density, (c) IP acquisition time vs. network fragmentation ratio, (d) IP acquisition time vs. length of IP passing (hops).

Fig. 15 shows the impacts of vehicle speed, vehicle density, network fragmentation ratio and length of IP passing to IP acquisition time. As the vehicle speed, vehicle density, network fragmentation ratio and length of IP passing increase, the IP acquisition time also increases because higher speed tolerates shorter handoff delay, higher vehicle density incurs higher contentions and collisions, higher network fragmentation ratio hinders more IP passing to be success, and longer length of IP passing incurs longer propagation delay. Among the three schemes, the IP acquisition time of our scheme is the lowest, follows by the IP passing scheme and MIPv6 scheme. Our scheme performs the best because our scheme can provide more chance than IP passing scheme does to make IP passing successfully. The MIPv6 scheme performs the worst because mostly it acquires the IP address from the DHCP server. The 
analysis results of our scheme are quite close to the simulation results, which indicates that our analysis is proper.

\section{B. IP lifetime}

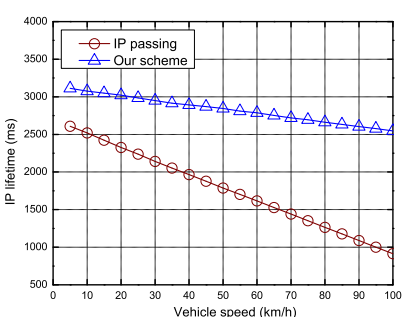

(a)

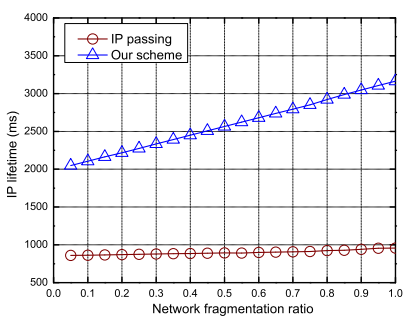

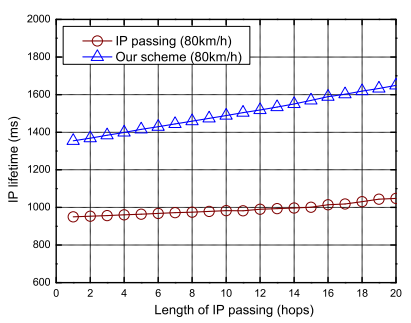

(d)

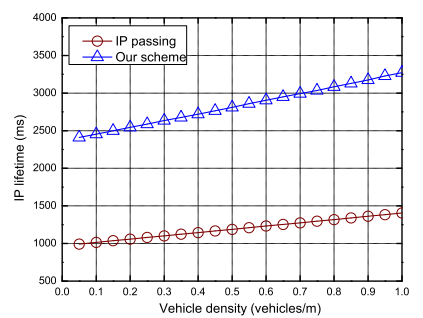

Fig. 16. (a) IP lifetime vs. vehicle speed, (b)IP lifetime vs. vehicle density, (c) IP lifetime vs. network fragmentation ratio, (d) IP lifetime vs. length of IP passing (hops).

Fig. 16 shows the impacts of vehicle speed, vehicle density, network fragmentation ratio and length of IP passing to IP lifetime. As the vehicle density, network fragmentation ratio and length of IP passing increases, the IP lifetime also increases. As the vehicle speed increases, the IP lifetime decreases. The IP lifetime of our scheme is longer than that of the IP passing scheme because in our scheme, even LMN cannot pass its IP address to EMN, it will still pass its IP address to the vehicle that remains in the target $\mathrm{BS}$ and thus postpones the time to release its IP to the DHCP server and extends IP lifetime. However, in the IP passing scheme, if LMN cannot pass its IP address to EMN, it will release its IP address to the DHCP server.

\section{Handoff latency}

Fig. 17 shows the impacts of vehicle speed, vehicle density, network fragmentation ratio and length of IP passing to handoff latency. Since the handoff latency is proportional to the IP acquisition time, the result is similar to that of the IP acquisition time. As the vehicle speed, vehicle density, network fragmentation ratio and length of IP passing increase, the handoff latency also increases. Among the three schemes, the handoff latency of our scheme is the lowest, follows by the IP passing scheme and MIPv6 scheme. Our scheme performs the best because our scheme has the lowest IP acquisition time; while MIPv6 has the highest IP acquisition time.

\section{Packet loss rate}

Fig. 18 shows the impacts of vehicle speed, vehicle density, network fragmentation ratio and length of IP passing to packet loss rate. As the vehicle speed, vehicle density, network fragmentation ratio and length of IP passing increases, the packet loss rate also increases. Among the three schemes, the packet loss rate of our scheme is the lowest, follows by the IP passing scheme and MIPv6 scheme. Our scheme performs the best because the handoff latency of our scheme is the

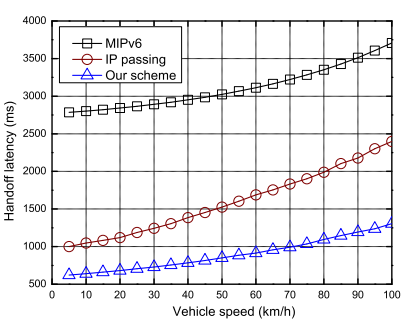

(a)

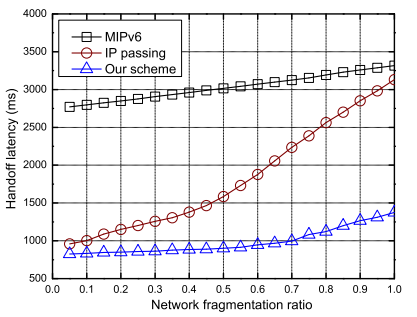

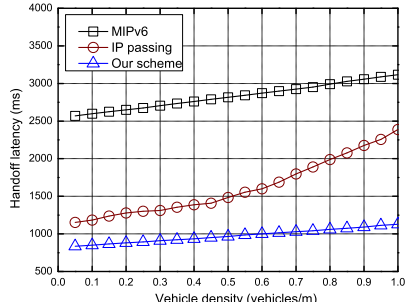

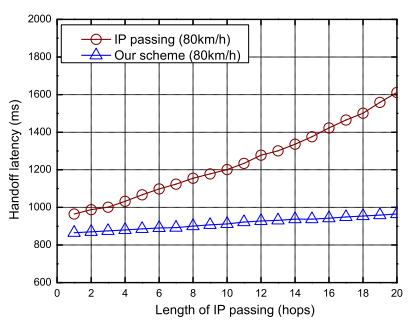

Fig. 17. (a) Handoff latency vs. vehicle speed, (b) Handoff latency vs. vehicle density, (c) Handoff latency vs. network fragmentation ratio, (d) Handoff latency vs. length of IP passing (hops).
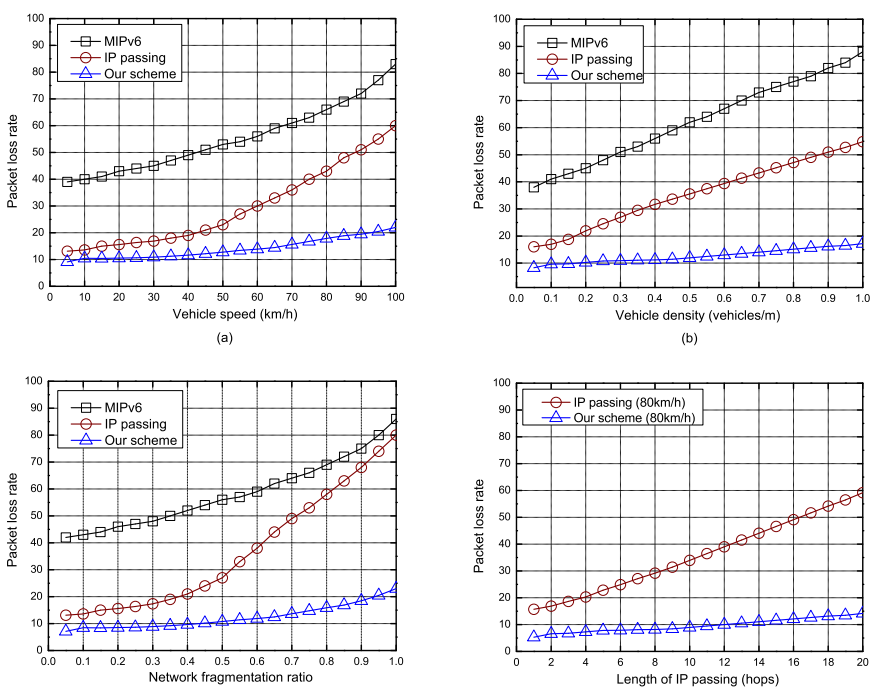

(c)

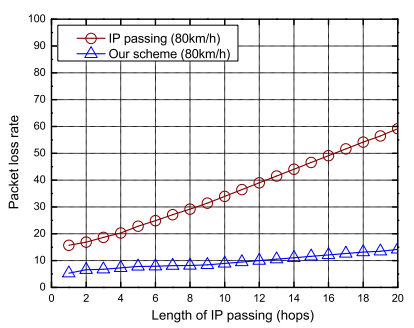

(d)

Fig. 18. (a) Packet loss rate vs. vehicle speed, (b) Packet loss rate vs. vehicle density, (c) Packet loss rate vs. network fragmentation ratio, (d) Packet loss rate vs. length of IP passing (hops).

shortest and thus its connection to Internet is the most stable and can achieve the lowest packet loss rate. The MIPv6 scheme performs the worst because its handoff latency is the longest and thus its connection to Internet is more likely to be broken and causes higher packet loss rate.

\section{E. Throughput}

Fig. 19 shows the impacts of vehicle speed, vehicle density, network fragmentation ratio and length of IP passing to throughput. As the vehicle speed, vehicle density, network fragmentation ratio and length of IP passing increases, the throughput decreases. Among the three schemes, the throughput of our scheme is the highest, follows by the IP passing scheme and MIPv6 scheme. As the handoff latency and packet loss rate increase, the throughput decreases. Our scheme performs the best because the handoff latency and packet loss 


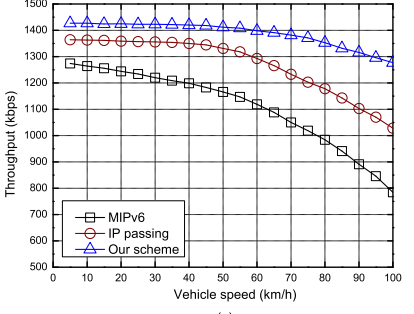

(a)

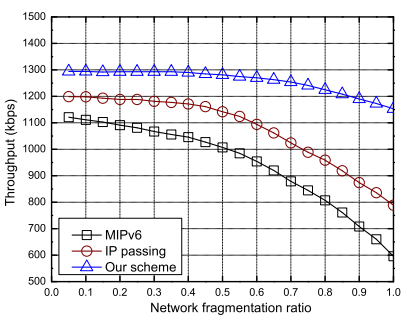

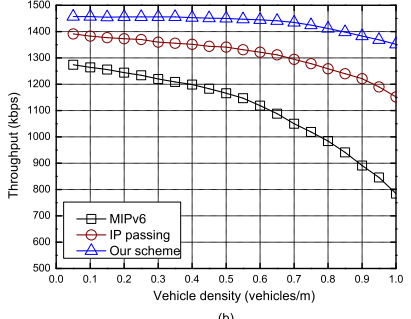

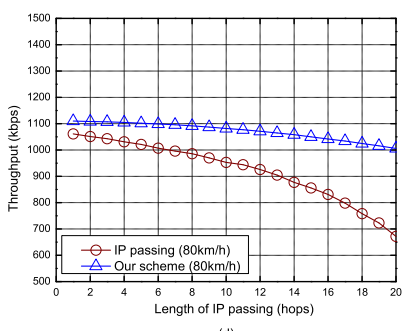

Fig. 19. (a) Throughput vs. vehicle speed, (b) Throughput vs. vehicle density, (c) Throughput vs. network fragmentation ratio, (d) Throughput vs. length of IP passing (hops).

rate of our scheme are the lowest; while the handoff latency and packet loss rate of the MIPv6 scheme is the highest.

\section{F. Message overhead}
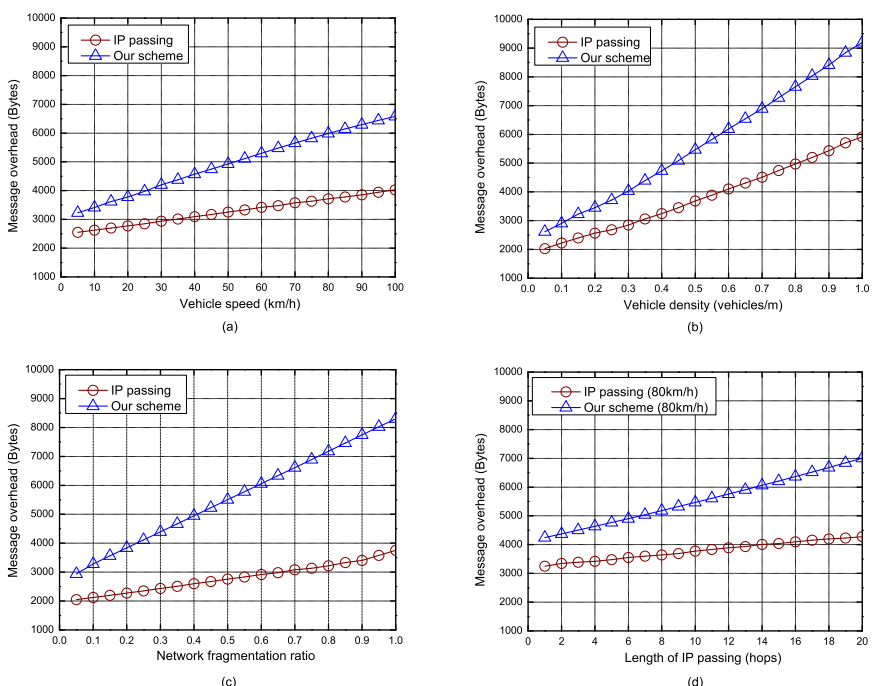

Fig. 20. (a) Message overhead vs. vehicle speed, (b) Message overhead vs. vehicle density, (c) Message overhead vs. network fragmentation ratio, (d) Message overhead vs. length of IP passing (hops).

Fig. 20 shows the impacts of vehicle speed, vehicle density, network fragmentation ratio and length of IP passing to message overhead. As the vehicle density, network fragmentation ratio and length of IP passing increases, the message overhead also increases. The message overhead of our scheme is higher than that of the IP passing scheme because in our scheme, even LMN cannot pass its IP address to EMN, it will still pass its IP address to the vehicle that remains in the target BS and thus incurs more message overhead. Besides, each vehicle needs to collect the information of the nearby vehicles.

\section{CONCLUSIONS}

In this paper, we have proposed an IP passing protocol for vehicular ad hoc networks with network fragmentation. Although IP passing may reduce handoff latency, but it cannot solve the network fragmentation problem because the IP address cannot be passed to the intended vehicle. In the proposed protocol, LMN will pass its IP address to the vehicle that remains in the target BS and thus postpones the time to release its IP to the DHCP server. During the extended IP lifetime, as a vehicle is going to enter the target $\mathrm{BS}$, it can acquire an IP address through multi-hop relays from the vehicle which carries the released IP, and thus it can reduce the handoff delay and maintain the connectivity to Internet. Simulation results have shown that the proposed scheme is able to reduce IP acquisition time, packet loss rate, and extend IP lifetime with extra message overhead.

\section{ACKNOWLEDGMENT}

This work was supported by grants NSC-97-2221-E-305003-MY3, NSC-99-2221-E-305-011, and NSC-99-2221-E128-002 from the National Science Council of the R.O.C.

\section{REFERENCES}

[1] C.Perkins, "Ip mobility support for ipv4," Internet Engineering Task Force (IETF), RFC-3344, Augst 2002.

[2] D. Johnson, C. Perkins, and J. Arkko, "Mobility support in ipv6," Internet Engineering Task Force (IETF), RFC-3775, June 2004.

[3] R. Koodli and Ed., "Fast handovers for mobile ipv6," Internet Engineering Task Force (IETF), RFC-4068, July 2005.

[4] S. Gundavelli, Ed., K. Leung, V. Devarapalli, K. Chowdhury, and B. Patil, "Proxy mobile ipv6," Internet Engineering Task Force (IETF), RFC-5213, Augest.

[5] H. Soliman, C. Castelluccia, K. E. Malki, and L. Bellier, "Hierarchical mobile ipv6 mobility management (hmipv6)," Internet Engineering Task Force (IETF), RFC-4140, Augest 2005.

[6] R. Droms, H. Packard, T. L. B. Volz, C. Perkins, and M. Carney, "Dynamic host configuration protocol for ipv6 (dhcpv6)," Internet Engineering Task Force (IETF), RFC-3315, July 2003.

[7] R. Droms, "Dynamic host configuration protocol," Internet Engineering Task Force (IETF), RFC-2131, March 1997.

[8] W. L. T Arnold and J. Zhao, "Ip address passing for vanets," IEEE International Conference on Pervasive Computing and Communications (PERCOM), Hong Kong, pp. 70-79, March 2007.

[9] M. Fazio, C. E, Palazzi, S. Das, and M. Gerlas, "Facilitating real-time applications in vanets through fast address auto-configuration," In the proceedings of 4th IEEE Consumer Communications and Networking Conference, pp. 981-985, Jan 2007.

[10] M. Fazio, C. E. Palazzi, S. Das, and M. Gerla, "Posters: Automatic ip address configuration in vanets," Proceedings of the 3rd international workshop on Vehicular ad hoc networks, pp. 100-101, September 2006.

[11] Mohandas, B.K., and R. Liscano, "Ip address configuration in vanet using centralized dhcp," Local Computer Networks (LCN), pp. 608-613, Oct. 2008.

[12] Y.-S. Chen, C.-H. Cheng, C.-S. Hsu, and G.-M. Chiu, "Network mobility protocol for vehicular ad hoc networks," Wireless Communications and Networking Conference (WCNC), pp. 1-6, April 2009.

[13] J. M. Lee, M. J. Yu, Y. H. Yoo, and S. G. Choi, "A new scheme of global mobility management for inter-vanets handover of vehicles in v2v/v2i network environments," Networked Computing and Advanced Information Management (NCM), pp. 114-119, Sept. 2008.

[14] K.-L. Chiu, R.-H. Hwang, and Y.-S. Chen, "Cross-layer design vehicleaided handover scheme in vanets," Wireless Communications and Mobile Computing (Published online), Oct. 2009.

[15] "The network simulator ns-2: http://hpds.ee.ncku.edu.tw/ smallko/ns2/ns2.htm." 